\title{
Three-Dimensional Structure and Defects in Colloidal Photonic Crystals Revealed by Tomographic Scanning Transmission X-ray Microscopy
}

\author{
Jan Hilhorst, ${ }^{\dagger}$ Matti M. van Schooneveld, ${ }^{\ddagger}$ Jian Wang, ${ }^{\S}$ Emiel de Smit, ${ }^{\ddagger}$ Tolek Tyliszczak, ${ }^{\|}$Jörg Raabe, ${ }^{\perp}$ \\ Adam P. Hitchcock, ${ }^{\S}$ Martin Obst, ${ }^{\#}$ Frank M. F. de Groot, ${ }^{\ddagger}$ and Andrei V. Petukhov*, ${ }^{\dagger}$ \\ ${ }^{\dagger}$ Van 't Hoff Laboratory for Physical and Colloid Chemistry, Debye Institute for Nanomaterials Science, Utrecht University, \\ Padualaan 8, $3584 \mathrm{CH}$ Utrecht, The Netherlands \\ ${ }^{\ddagger}$ Inorganic Chemistry and Catalysis, Debye Institute for Nanomaterials Science, Utrecht University, Sorbonnelaan 16, 3584 CA \\ Utrecht, The Netherlands \\ ${ }^{\S}$ Canadian Light Source, University of Saskatchewan, Saskatoon, Saskatchewan S7N OX4, Canada \\ "Advanced Light Source, Lawrence Berkeley National Laboratory, Berkeley, California 94720, United States \\ ${ }^{\perp}$ Swiss Light Source, Paul Scherrer Institute, 5232 Villigen, Switzerland \\ ${ }^{\#}$ Center for Applied Geoscience, Eberhard Karls Universität Tübingen, Sigwartstrasse 10, 72076 Tübingen, Germany
}

Supporting Information

ABSTRACT: Self-assembled colloidal crystals have attracted major attention because of their potential as low-cost threedimensional (3D) photonic crystals. Although a high degree of perfection is crucial for the properties of these materials, little is known about their exact structure and internal defects. In this study, we use tomographic scanning transmission X-ray microscopy (STXM) to access the internal structure of selfassembled colloidal photonic crystals with high spatial resolution in three dimensions for the first time. The positions of individual particles of $236 \mathrm{~nm}$ in diameter are identified in three dimensions, and the local crystal structure is revealed. Through image analysis, structural defects, such as vacancies and stacking faults, are identified. Tomographic STXM is shown to be an attractive and complementary imaging tool for photonic materials and other strongly absorbing or scattering materials that cannot be characterized by either transmission or scanning electron microscopy or optical nanoscopy.

\section{INTRODUCTION}

Photonic crystals are currently under research for their potential in a wide variety of applications. Their capability to manipulate the propagation of light opens the way to the perfecting of existing technology, such as optical fibers ${ }^{1,2}$ or lasers, ${ }^{3}$ but also to exotic new devices, such as optical chips. ${ }^{4}$

Several methods exist to obtain crystals with a full photonic band gap in the visible spectrum. Lithographic methods have been shown to be very effective for producing high-quality materials with any desired structure, ${ }^{5}$ even including defect regions that can be used as circuits to build all-optical logical elements. $^{6}$ The applicability of such structures, however, is limited by slow and costly production processes.

Self-assembly of the right building blocks into large photonic crystals is more promising in terms of speed and production cost. $^{7,8}$ This technique has other drawbacks, mainly in the intrinsic disorder present in such systems ${ }^{9-12}$ and the difficulty of including functional defects at desired positions. ${ }^{13}$ Although it has been shown that selective incorporation of defects is possible, defect growth resulted in a decrease in the degree of perfection of crystals ${ }^{14}$ or required laborious post-processing of highly perfect crystals. ${ }^{15}$ Because of the small energy difference between the hexagonal close-packed (hcp) and face-centered cubic (fcc) crystal structures, ${ }^{16}$ self-assembly often results in a mixture of the two structures, which is unfavorable for the optical properties. The most promising growth process in this category is convective assembly. ${ }^{7,8,17}$ This method produces good quality crystals with a mainly fcc structure, ${ }^{11,17}$ which is required for producing materials with a full photonic band gap in the visible spectrum.

Despite the large interest in these structures, much is still unknown about the processes governing self-assembly, driving the crystal into its fcc structure. In addition, most of what is known about the crystal structure is obtained through either

Received: December 1, 2011

Revised: January 16, 2012

Published: January 19, 2012 
surface methods, such as scanning electron microscopy (SEM), ${ }^{7,9,17}$ or bulk averaging methods, such as microradian $\mathrm{X}$-ray diffraction ${ }^{11,18,19}$ or field-stop filtered microscopy. ${ }^{20}$ SEM has the disadvantage that no information about the internal crystal structure is obtained and it can only be used as a rough guide to the quality of a crystal. Bulk averaging techniques provide information about stacking sequences over large areas of crystal but neglect the local defect structure, which is so important for the optical properties ${ }^{21}$ and for the growth process. $^{12,22}$

To fully understand the relations between the growth process and the resulting structure, detailed knowledge about the local internal structure has to be obtained. Unfortunately, this is not straightforward. Several studies exist that address the local structure, but the methods used are not widely applicable. ${ }^{9,22}$ A powerful technique for studying the local structure in three dimensions is confocal microscopy. ${ }^{22-24}$ Since the advent of optical microscopic techniques breaking the diffraction limit, $^{25,26}$ similar techniques have reached a resolution that is sufficient to study most colloidal systems. However, these techniques require the use of dyed particles and, more importantly, the use of systems that do not strongly absorb or scatter visible light. In the case of convectively assembled crystals, scattering has to be prevented by infiltrating the crystal with a refractive index matching liquid. ${ }^{24}$ The same capillary forces that play a role during crystal growth are applied again during infiltration, possibly altering the grown crystal structure. In addition, because the colloidal fcc structure does not possess a full photonic band gap, the crystal structure has to be "inverted". This involves the infiltration of the pores in the crystal with a high refractive index material and a subsequent removal of the original spheres. ${ }^{7,27}$ In this process, the refractive index contrast in the material is maximized. Therefore, no suitable refractive index matching fluids are available, and visible light microscopy of these structures is not possible.

A technique that has proven valuable for the characterization of the internal structure of nanocrystal solids is tomographic transmission electron microscopy. Several studies exist that address the internal structure of crystals made of semiconductor quantum dots and magnetic and metallic nanoparticles. ${ }^{28,29}$ However, these materials have a thickness of several tens of nanometers, while photonic structures are usually over a few micrometers thick. The strong attenuation of an electron beam by such thick structures prohibits the use of this technique here.

Recent developments in X-ray imaging have yielded a spectrum of techniques with the potential to access the internal structure of colloidal photonic crystals. Among these are coherent diffraction imaging (CXDI), ${ }^{30}$ ptychography, ${ }^{31}$ and high-resolution transmission hard X-ray microscopy (HRTXM) ${ }^{32}$ The relatively high penetration power of X-rays enables studies of almost any material and thickness. In CXDI and ptychography, use is made of phase retrieval algorithms to reconstruct a real-space representation from diffraction patterns. The merits of ptychography were demonstrated in a tomographic reconstruction of a bone sample ${ }^{33}$ with a voxel size as small as $65 \mathrm{~nm}$ in all three dimensions. Full-field HRTXM imaging of colloidal crystals was recently demonstrated at $12 \mathrm{keV}$ photon energy. ${ }^{32}$ At such a high energy, a large penetration depth of the probing beam can be achieved with a spatial resolution of $\sim 100 \mathrm{~nm}$. However, phase contrast is much larger than absorption contrast if the photon energy is not tuned to a specimen absorption edge. For this reason, interpretation of resulting data remains challenging.
To address the local crystal structure, we have recently shown that, in two dimensions, superior resolution as good as $30 \mathrm{~nm}$ can be obtained by scanning transmission X-ray microscopy (STXM) imaging. ${ }^{34}$ The major benefit of this technique over other $\mathrm{X}$-ray techniques is the possibility to directly obtain element-specific absorption contrast, significantly simplifying data analysis. A detailed comparison between optical microscopy methods, ${ }^{23-26}$ SEM, and STXM was given in our earlier paper. ${ }^{34}$

For crystals thicker than three hexagonal layers, twodimensional (2D) imaging cannot provide conclusive information about the internal structure. For example, four-layer stacking sequences $\mathrm{ABCA}, \mathrm{ACBA}, \mathrm{ABAC}$, and $\mathrm{ACAB}$ all provide the same $2 \mathrm{D}$ profile. We have therefore extended the use of STXM to include tomographic reconstruction, enabling studies of the internal three-dimensional (3D) crystal structure of thicker crystals, including their internal defect structure. For these particular structures, tomographic STXM is the only method that can provide this type of information for reasons described above. The crystals, grown from silica particles of 236 $\mathrm{nm}$ in diameter, were measured at and before their silicon $\mathrm{K}$ edge, at 1845 and $1830 \mathrm{eV}$, respectively, to obtain pure material absorption contrast and, therefore, information on sample thickness. $^{34}$

\section{EXPERIMENTAL SECTION}

Synthesis of Silica Colloids and Their Crystals. Silica particles were synthesized according to the method described by Stöber et al. ${ }^{35}$ A coating of 3-(trimethoxysilyl)propyl methacrylate (TPM) was added following the method by Philipse and Vrij. ${ }^{36}$ The particle diameter was determined using transmission electron microscopy (TEM) and was found to be $236 \mathrm{~nm}$ with a relative standard deviation of $5 \%$. This size was verified for particles in solution using dynamic light scattering.

Crystals were grown onto various types of substrates by immersing the substrate in an aqueous dispersion (Millipore water, $18 \mathrm{M} \Omega \mathrm{cm}$ ) with particle concentrations ranging from 0.1 to $1.0 \%$ (v/v) and evaporating the solvent at elevated temperatures without gas flow. ${ }^{17} \mathrm{~A}$ schematic of the growth process is shown in Figure 1a. We employed $100 \mathrm{~nm}$ thick $\mathrm{Si}_{3} \mathrm{~N}_{4}$ windows (Silson, Ltd.) as well as cut TEM grids coated with a $2-3 \mathrm{~nm}$ thick layer of platinum or palladium (Ted Pella, Substratek) as substrates. These were attached to a glass slide and added to a crystal growth dispersion under an slide inclination varying from $0^{\circ}$ to $30^{\circ}$ with respect to the vertical direction. The crystal used for the tomographic reconstruction in Figures 3 and 4 was grown from a $0.1 \mathrm{vol} \%$ aqueous dispersion under a substrate inclination of $0^{\circ}$ at a temperature of $70{ }^{\circ} \mathrm{C}$.

STXM Imaging and Data Processing. STXM measurements were performed at the 10ID-1 soft X-ray spectromicroscopy beamline at the Canadian Light Source (CLS) in Saskatoon, Saskatchewan, Canada. ${ }^{37}$

A schematic of the setup is displayed in Figure 2a. In short, monochromatic X-rays with an energy between 130 and $2500 \mathrm{eV}$ are focused onto a spot on the sample by a Fresnel zone plate. For the silica particles described in this paper, we measured at photon energies of 1845 and $1830 \mathrm{eV}$, i.e., above the silicon $\mathrm{K}$ edge and in the pre-edge. Measured transmission is converted to optical density (OD) through the Lambert-Beer law. Background images recorded at $1830 \mathrm{eV}$ are subtracted from the $1845 \mathrm{eV}$ edge images, obtaining pure silicon absorption contrast. The lower size limit of the beam spot is determined by the quality of the zone plate, the outermost zone width, and the X-ray energy. In the experiment described here, the instrument spatial resolution was $30 \mathrm{~nm}$. Higher order focal points of the zone plate and zero-order light are filtered out by the order-sorting aperture (OSA) and a central stop on the zone plate. Because of the relatively long focal length of the zone plate at the energies used in this experiment, the depth of focus of the beam $(\sim 3700 \mathrm{~nm})$ was of the same order of magnitude as the whole thickness of the sample, 
ensuring optimum resolution in the whole of the crystal. ${ }^{34}$ Finally, a detector consisting of a scintillator and a photomultiplier tube detects the transmitted X-ray intensity. A piezoelectric sample stage is used to scan the sample along the plane normal and parallel to the beam, gathering a focused transmission image of the desired region in the sample.

Prior to tomographic reconstruction, all images were processed using the aXis 2000 software package. ${ }^{38}$ For all images measured above the silicon $\mathrm{K}$ edge, a pre-edge image measured at an energy just below the onset of the edge was subtracted to obtain pure silicon absorption contrast. Tomographic reconstruction was performed with the IMOD tomography package. ${ }^{39}$ This package was originally designed for TEM data sets but is able to process any set of grayscale tiff files. The package was used for further alignment of the data set images and subsequent tomographic reconstruction through a weighted backprojection algorithm.

\section{RESULTS AND DISCUSSION}

Colloidal crystals were grown via convective assembly ${ }^{17}$ on 100 $\mathrm{nm}$ thick silicon nitride windows or on cut TEM grids, as discussed in the Experimental Section. A schematic of the growth method is shown in Figure 1a. The reason for growing crystals on such thin substrates is the limited penetration depth of soft X-rays. At the energies used here, $1830-1845 \mathrm{eV}$, this can be up to several micrometers. Because the substrate should not attenuate the beam too much, it should at most be $1 \mu \mathrm{m}$ thick. Typical SEM images of crystals grown on thicker glass slides are displayed in panels $b$ and $c$ of Figure 1 . In these images and in all images in this paper, the growth direction was from top to bottom.

The growth conditions on such small slides of material, especially the cut TEM grids, may be significantly different from those on a larger substrate. As seen in Figure $1 b$, the rows of touching particles are aligned neither horizontally nor vertically. This is the result of the curvature of the liquid meniscus near the edge of a substrate. A similar effect can be observed in the region used for tomographic reconstruction. The exact effect of this curvature on the crystal growth mechanism and the concentration of defects is unknown, because studies of the internal crystal structure have been scarce up until this moment.

For characterization of the internal structure of the crystals, the STXM setup described in the Experimental Section was used. The choice of X-ray microscope is of paramount importance for the planning of an experiment. Although in this particular experiment a scanning transmission X-ray microscope was used, the use of full-field transmission X-ray microscopes (TXMs) would be beneficial in terms of image acquisition speed, enabling, for example, an extension of the data set to include more angles or a longer integration time to obtain the signal at angles larger than the $60^{\circ}$ reported here. In addition to acquisition speed (TXM favorable), microscope selection criteria include radiation damage (STXM favorable), energy resolution (STXM favorable), and available energy range (differs for each setup, only necessary if absorption contrast is desired). These criteria have to be carefully weighed to ensure optimal data acquisition.

A schematic representation of the STXM setup is given in Figure 2a. In short, monochromatic X-rays are focused onto a $\sim 30 \mathrm{~nm}$ spot on the sample, where transmission is measured. When the sample is scanned through the focal point, a transmission image is obtained. For a more detailed description of the technique, we refer to the Experimental Section. An example of a STXM image of a crystal consisting of $492 \mathrm{~nm}$
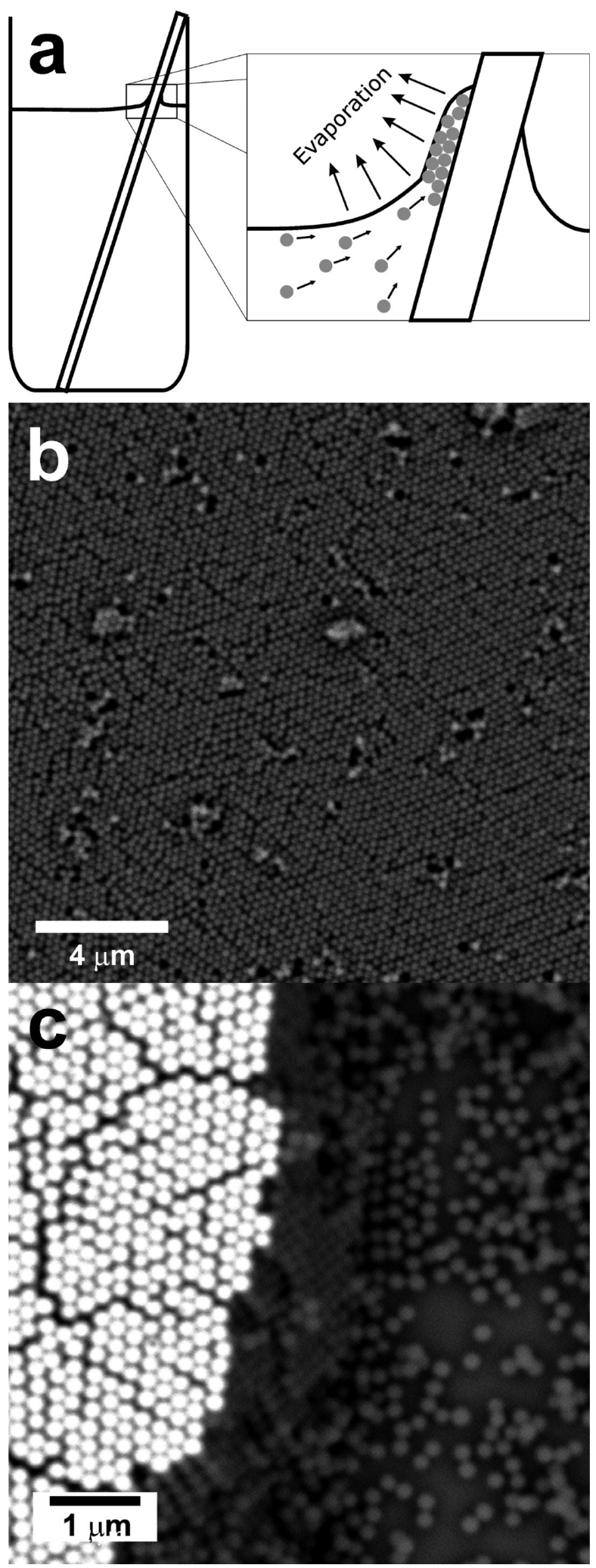

Figure 1. (a) Schematic representation of the crystal growth by convective assembly is shown. Evaporation of the solvent at the curved meniscus and the crystal surface creates a flow of particles toward the growing crystal. (b and c) Representative SEM images of the crystals obtained on glass substrates.

particles on a silicon nitride window is given in Figure $2 \mathrm{~b}$. The image has been converted from transmission to OD via the Lambert-Beer law, to provide a more insightful linear thickness 


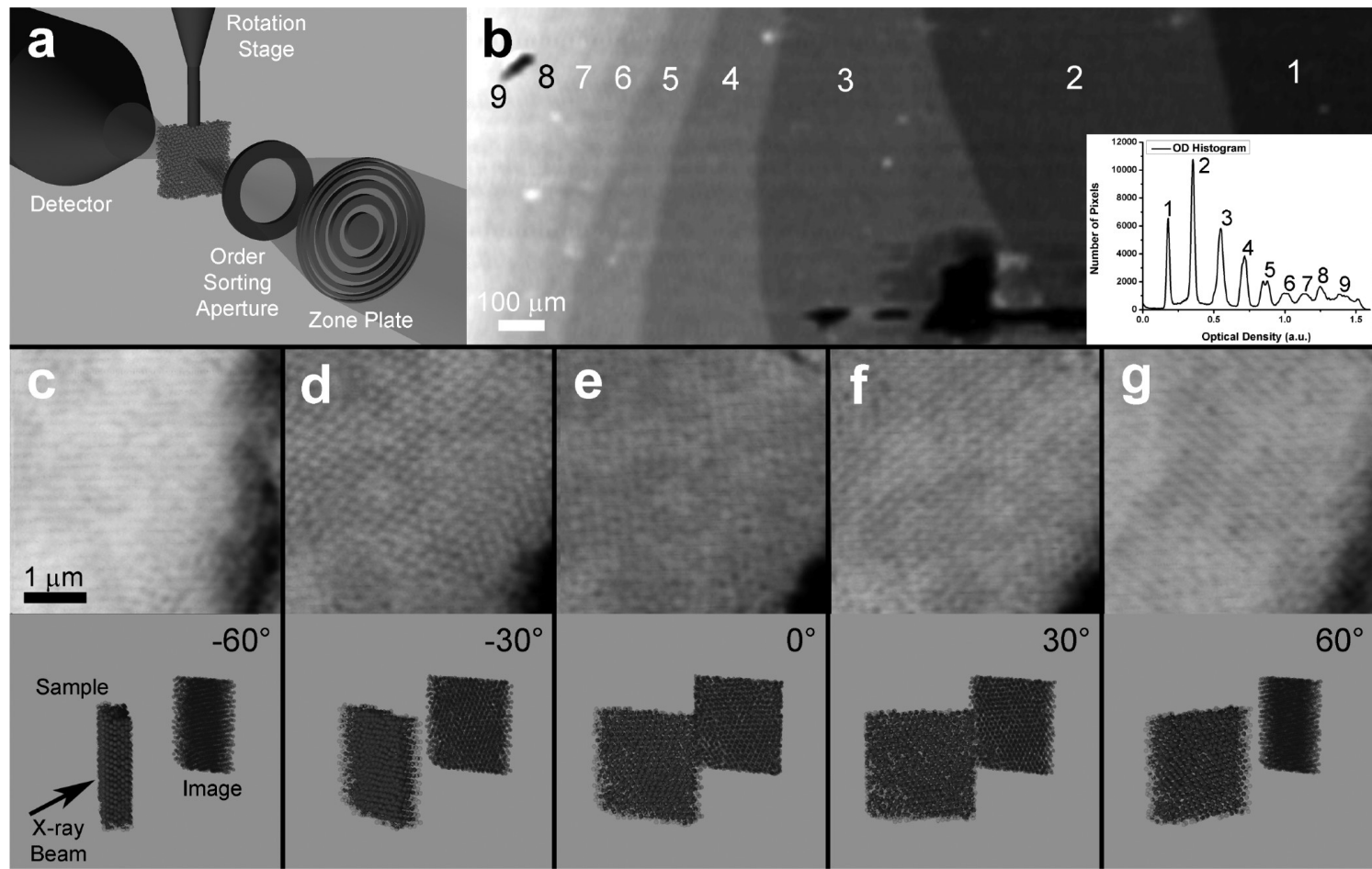

Figure 2. (a) Schematic representation of the microscopy setup as described in the text. (b) A $2.0 \times 0.8 \mathrm{~mm}^{2}$ STXM false-color overview of a crystal that is up to nine layers thick. Different colors represent different thicknesses, and thickness increases from right to left. Individual particles cannot be distinguished at this magnification. The inset shows an intensity histogram, identifying up to nine particle layers. ( $c-g$ ) OD images obtained from STXM measurements on a real crystal. The angles at which the scans were made are given below each image, along with a schematic indicating the effect of the measurement angle on transmission and width of the ROI.

scale. ${ }^{34}$ From right to left, sharp, stepwise changes in color indicate transitions toward a thicker crystal. A histogram of the measured intensity distribution is shown in the inset. Every peak corresponds to a certain thickness of crystal, with the highest measured intensity (right) corresponding to the thinnest piece of crystal. This way, up to nine layers of material can be identified, as expressed by the numbers in the histogram. The corresponding numbers have been added to the OD image as well.

Overviews like Figure $2 \mathrm{~b}$ are suitable for selecting regions of interest (ROIs) in a sample or for investigating the transmission as a function of material thickness. To be able to reconstruct crystal structures on a single-particle level, however, more detailed images have to be obtained. Panels $\mathrm{c}-\mathrm{g}$ of Figure 2 show such images, with a field of view of $5 \times 5 \mu \mathrm{m}^{2}$ and a pixel size of $25 \times 25 \mathrm{~nm}^{2}$. Particles in the images are $236 \mathrm{~nm}$ in diameter. By measuring such a ROI at different angles with respect to the beam, a rotation series is obtained through which the $3 \mathrm{D}$ crystal structure can be reconstructed. To allow for sample rotation at short working distances between the focusing optics and the detector, samples were prepared in a way similar to the method described by Obst et al. ${ }^{40}$ In short, Pd- and Pt-coated TEM grids were cut on two sides to leave a small ribbon of material approximately $1 \mathrm{~mm}$ wide onto which the crystal was grown. This ribbon was glued to the rotation stage described by Johansson et al. ${ }^{41}$ Suitable regions for tomogram recording were selected on the basis of three criteria. First, sample thickness had to be large enough to obtain relevant information about the internal crystal structure but small enough to avoid signal saturation at high scanning angles. Second, regions close to the edge of a grid square had to be avoided because the grid edges would interfere with imaging upon rotation. Finally, to ensure proper sample positioning after every rotation step, a region had to contain a prominent feature, such as a crystal crack. The latter condition may be avoided by adding specific markers to a crystal to make any region recognizable, such as core-shell particles with a highdensity core or a small concentration of gold nanoparticles. In this case, care has to be taken not to influence the crystal structure in any post-processing step.

Pictures were taken over a range from $-60^{\circ}$ to $60^{\circ}$ with steps of $4^{\circ}$. At larger angles, the signal was too attenuated to obtain reliable images. Every third step, an extra angle was recorded after a $2^{\circ}$ interval to increase the size of the data set and, with that, the resolution of the tomographic reconstruction.

Panels $\mathrm{c}-\mathrm{g}$ of Figure 2 show the region used for tomographic reconstruction recorded from five different angles. Below each image, a schematic representation of the sample and its image is provided for clarity. A movie showing the full rotation series covering all measured angles is provided in the Supporting Information. In the bottom right corner of each image, the crack that has been used as a reference for sample positioning can be seen. The projections have been converted from transmission to OD images. From a calibration image, a single layer was found to have a maximum OD of approximately 0.25 . Considering a packing fraction of $74 \%$ for close packed spheres, the average OD of 1.5 measured at normal incidence corresponds to approximately eight layers of particles. Intensity modulations corresponding to periodicities in the crystal lattice could be observed in almost all projections and are attributed to alignment of particles along specific crystallographic directions. In total, 41 projections were used for tomographic reconstruction. 


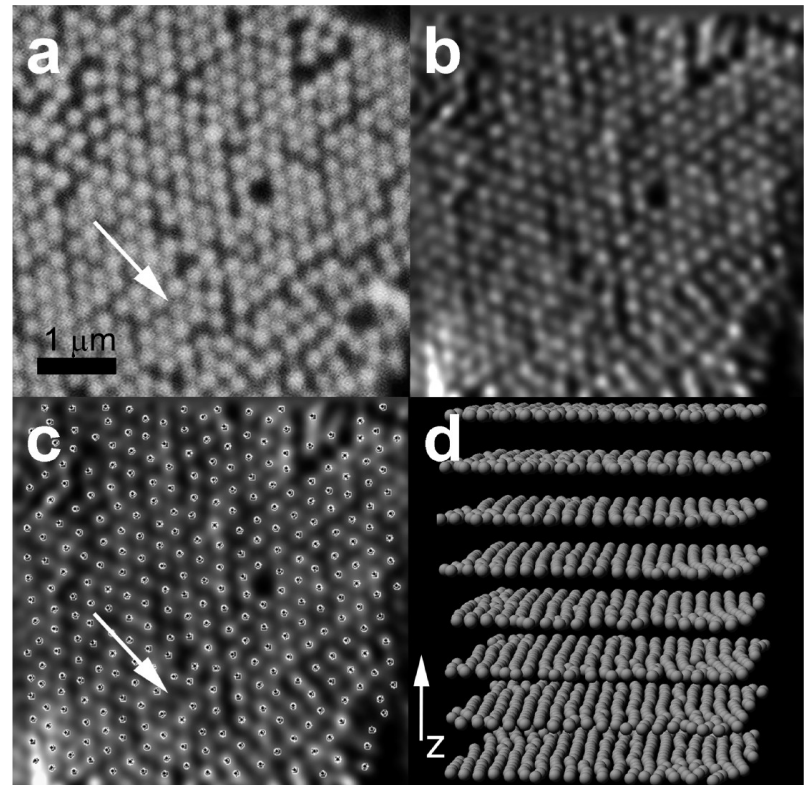

Figure 3. $(a-c)$ Top layer of the crystal in SEM view, tomographic STXM reconstruction, and detected particle positions are shown, respectively. Arrows indicate the only particle that was not identified by the detection algorithm. (d) Model view showing all detected particles in the eight layers of the reconstruction. The $z$ axis has been stretched for clarity.

Results of the tomographic reconstruction are displayed in Figure 3. Figure 3a contains an SEM image of the ROI, and Figure $3 \mathrm{~b}$ depicts the same layer selected from the reconstruction of the STXM tomography data set. The thickness of this tomographic slice is approximately $150 \mathrm{~nm}$, corresponding to the resolution in the out-of-plane direction. From these images, the perfect agreement between direct imaging and tomographic reconstruction is apparent. Only a slight discrepancy can be observed at the top edge and the bottom left corner of the reconstruction. These artifacts are attributed to misalignments during acquisition, resulting in the absence of these specific regions in some of the images. In total, the reconstruction produced a crystal of eight layers thick, in good agreement with the value calculated from the average OD. In addition, a disordered, open layer was also found at the bottom of the crystal (see Figure S1a of the Supporting Information). This layer consists of particles deposited on the back side of the TEM grid during crystal growth, as confirmed by the SEM image in Figure $S 1 b$ of the Supporting Information.

Particles and their positions were detected and digitized using an algorithm similar to the algorithm described by Crocker and Grier, ${ }^{42}$ written for Interactive Data Language (IDL). As seen in Figure 3c, the algorithm accurately detects the center of virtually every particle. Only one particle that is present in the SEM image has been missed, as indicated by the arrows in panels a and c of Figure 3. Also, in the region close to the crack, some discrepancies can be noted. Here, particles may have been slightly above or below the tomographic slice or excluded from some projection angles because of misalignments. In the aforementioned regions at the top edge and bottom left corner, some particles have been missed as well because of misalignment between measurements at different angles. Nonetheless, because over 300 particles were identified in the first layer, the detection was over 95\% accurate. A 3D rendering of the particles from all eight layers of the reconstruction is shown in Figure $3 \mathrm{~d}$. The disordered layer on the back of the grid has been left out for clarity.

Using the detected particle coordinates, the crystalline environment of every individual particle could be determined. To be able to detect the stacking of individual particles, an algorithm was used that correlates the positions of the nearest neighbors in the layer above and below each particle to each other. ${ }^{10}$ In hcp stacking, these have the same orientation (ABA stacking of hexagonal layers), while in fcc, the triangles they form are rotated $60^{\circ}$ with respect to each other (ABC

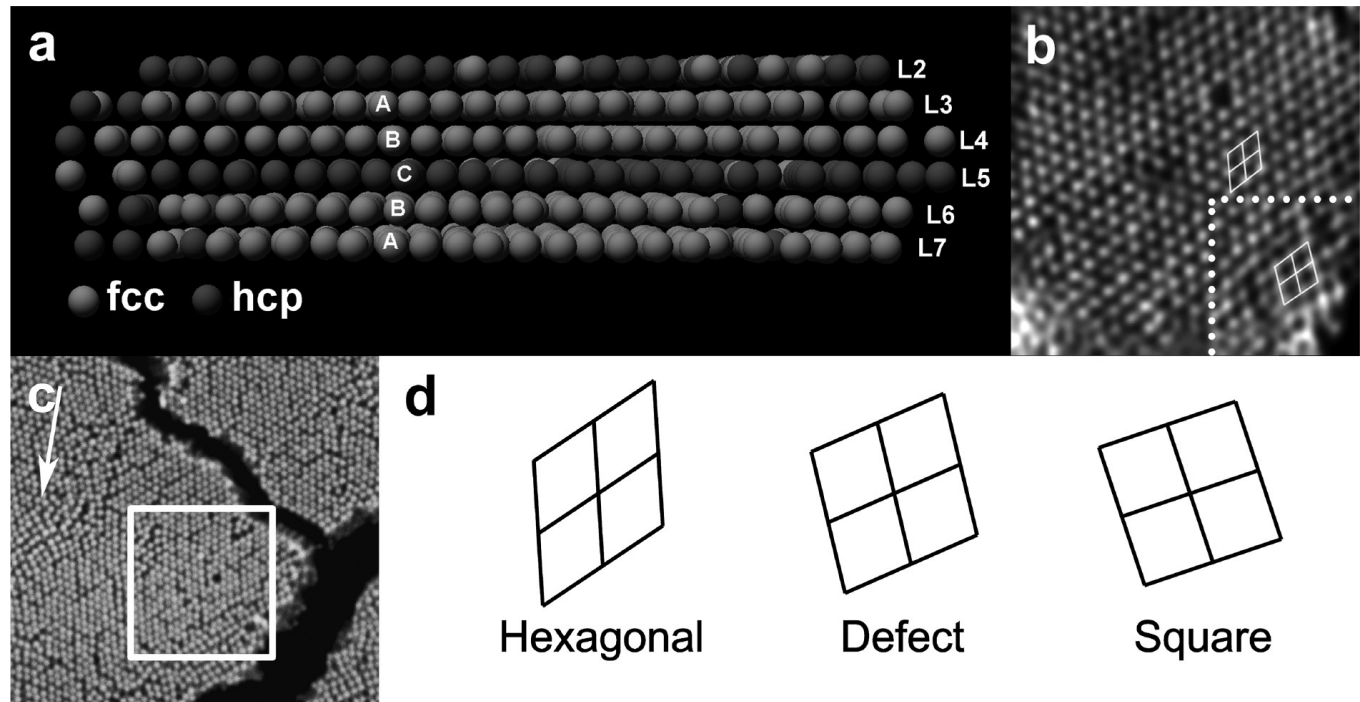

Figure 4. (a) Side view of the stacking sequence of layers 2 (top) to 7 (bottom) of the tomographic reconstruction is shown. Layers 1 and 8 are excluded because these have neither fcc nor hcp configuration. Both layers 2 and 5 are in hcp configuration and cause a transition of the crystal stacking from $\mathrm{ABC}$ to $\mathrm{CBA}$. Panel $\mathrm{b}$ shows a reconstruction of the fifth layer of the crystal, including the deformed region in the bottom right, which is contained in the dotted square. From the white overlaid lines, it is clear that this region is between square and hexagonal orientation, as illustrated in panel d. (c) Deformed region of the crystal outside of the tomographic ROI (highlighted by the white square) is indicated by the arrow. 
stacking). Figure $4 \mathrm{a}$ shows that the measured structure is dominated by fcc stacking, as expected for a convectively assembled colloidal crystal. ${ }^{17}$ However, the hcp stacking found in the fifth layer causes twinning from $A B C$ to $C B A$ in the middle of the crystal, which is unfavorable for the photonic band structure. ${ }^{21,43}$

Apart from stacking disorder, other local defects, such as vacancies, were observed. These are present in images of the first and fifth crystal layers displayed in Figures $3 \mathrm{~b}$ and $4 \mathrm{~b}$, respectively. The top right corner in Figure $3 \mathrm{~b}$ shows a triangle of line defects, as discussed by Meijer et al. ${ }^{10}$ This structure can be formed when a group of three vacancies aligns and three neighboring particles move from an $\mathrm{A}$ to a $\mathrm{B}$ position on the hexagonal plane below. It has been shown recently ${ }^{12}$ that defects like this may have a significant influence on the final crystal structure. However, we did not find similar defects in the inner structure of the studied crystal. The occurrence in the top layer may therefore be a surface effect, and further study is required to conclusively establish whether or not these defects occur more frequently.

The bottom right corner in panels a and b of Figure 3 shows a region of particles in square and rectangular configurations. These look similar to the transition regions that have been discussed by Meng et al. ${ }^{22}$ as possible candidates for causing the strong preference for fcc growth, albeit for thin crystals. The occurrence of these regions here could point to a similar mechanism for thicker crystals but conflicts with our observation of a stacking fault in the middle of the crystal. In a SEM overview image (Figure 4c), the region can be seen to extend over a longer range and a similar structure runs parallel to it, indicating an additional possible thickness transition. As mentioned earlier, however, SEM cannot provide us with any information on the deeper, internal crystal structure. We therefore investigated the same region in the tomographic reconstruction, with the advantage of being able to characterize the inside of the crystal. In Figure $4 b$, the fifth layer of the crystal is displayed. The bottom right region in this picture, contained in the dotted box, lies at the same position as the deformed region in panels $a$ and $b$ of Figure 3. It does not have square coordination, such as the first layer, but is not perfectly hexagonal either, as indicated by the drawn white lines connecting the particles and illustrated in Figure 4d. Although the structures found in the layers in between do not resemble those found by Meng et al., ${ }^{22}$ they are different from the rest of the crystal. For layers beyond the fifth layer, the corner returns to a perfect hexagonal orientation in line with the rest of the crystal. From this, it can be concluded that the deformed regions observed in the top layer with SEM extend several layers into the crystal but with a finite depth. It is therefore suspected that, if these regions do influence the structure as in ref 22 , they only influence the top layers. In this light, it is remarkable that we find a twinning plane at exactly the depth where the influence of the deformed region ends. The limited size of the deformed region in this reconstruction and the fact that the crystals were grown onto thin ribbons of material with curved menisci, however, do not allow us to draw further solid conclusions about the growth mechanism.

Nonetheless, the detail with which tomographic STXM yields information on the internal structure of convectively assembled crystals is unrivaled. For the first time, the defect structure of crystals grown from large crystals (>200 nm diameter) could be imaged at the single-particle level without restrictions in terms of refractive index mismatch, dyeing particles, or particle size.

For further experiments, the start-up of new X-ray microscopes (both STXM and TXM) with photon energies up to 14 $\mathrm{keV}$ promises microscopy with absorption contrast on virtually any element. With this, the imaging of thick inverted crystal structures (crystals of air spheres in high refractive index materials) consisting of heavier elements, such as titanium, ${ }^{27}$ iron, nickel, ${ }^{44}$ or even cadmium, ${ }^{45}$ becomes feasible. Also, the availability of both soft and hard X-ray microscopes might be used for the real-space study of colloidal liquid crystals ${ }^{46}$ (see Figure S2 of the Supporting Information for transmission properties of these compounds and several heavy metals). Furthermore, combinations of STXM with coherent diffraction imaging ${ }^{47}$ show promise of pushing the resolution into the sub$10 \mathrm{~nm}$ regime.

\section{CONCLUSION}

In summary, we have shown the applicability of absorption contrast soft X-ray tomography for the 3D imaging of crystals of inorganic colloids on a single-particle level. In a volume of 5 $\times 5 \times 2 \mu \mathrm{m}^{3}$, the positions and crystal structure of individual $236 \mathrm{~nm}$ particles were determined. Several types of crystal defects were identified, warranting further study into their role in the crystal growth process. Newly commissioning microscopes show promise of faster recording of high-quality data on virtually any material with element-specific absorption contrast. With these improvements in mind, we see X-ray tomography as a valuable addition to the characterization toolbox for photonic crystals in specific or condensed matter physics in general.

\section{ASSOCIATED CONTENT}

\section{Supporting Information}

Tomographic slice showing a disordered layer of colloids and SEM image indicating that this is located at the back of the substrate; calculated transmission spectra of several inorganic materials; and movie containing the full data set used for tomographic reconstruction. This material is available free of charge via the Internet at http://pubs.acs.org.

\section{AUTHOR INFORMATION}

\section{Corresponding Author}

*E-mail: a.v.petukhov@uu.nl.

\section{Notes}

The authors declare no competing financial interest.

\section{ACKNOWLEDGMENTS}

This work was financially supported by a VICI grant (FMFdG) of The Netherlands Organization for Scientific Research (NWO-CW). We thank beamline 10ID-1 (SM) at the CLS for beam time and support. The ALS is supported by the Director, Office of Science, Office of Basic Energy Sciences, of the U.S. Department of Energy, under Contract DE-AC0205CH11231. The CLS is supported by the Natural Sciences and Engineering Research Council of Canada, the National Research Council Canada, the Canadian Institutes of Health Research, the Province of Saskatchewan, Western Economic Diversification Canada, and the University of Saskatchewan.

\section{REFERENCES}

(1) Russell, P. Science 2003, 299, 358-362.

(2) Knight, J. C. Nature 2003, 424, 847-851. 
(3) Painter, O.; Lee, R. K.; Scherer, A.; Yariv, A.; O’Brien, J. D.; Dapkus, P. D.; Kim, I. Science 1999, 284, 1819-1821.

(4) Soljacic, M.; Luo, C.; Joannopoulos, J. D.; Fan, S. Opt. Lett. 2003, 28, 637-639.

(5) Lin, S. Y.; Fleming, J. G.; Hetherington, D. L.; Smith, B. K.; Biswas, R.; Ho, K. M.; Sigalas, M. M.; Zubrzycki, W.; Kurtz, S. R.; Bur, J. Nature 1998, 394, 251-253.

(6) Noda, S.; Tomoda, K.; Yamamoto, N.; Chutinan, A. Science 2000, $289,604-606$.

(7) Blanco, A.; Chomski, E.; Grabtchak, S.; Ibisate, M.; John, S.; Leonard, S. W.; Lopez, C.; Meseguer, F.; Miguez, H.; Mondia, J. P.; Ozin, G. A.; Toader, O.; Van Driel, H. M. Nature 2000, 405, 437-440.

(8) Vlasov, Y. A.; Bo, X. Z.; Sturm, J. C.; Norris, D. J. Nature 2001, 414, 289-293.

(9) Vlasov, Y. A.; Astratov, V. N.; Baryshev, A. V.; Kaplyanskii, A. A.; Karimov, O. Z.; Limonov, M. F. Phys. Rev. E: Stat. Phys., Plasmas, Fluids, Relat. Interdiscip. Top. 2000, 61, 5784-5793.

(10) Meijer, J. M.; De Villeneuve, V. W. A.; Petukhov, A. V. Langmuir 2007, 23, 3554-3560.

(11) Hilhorst, J.; Abramova, V. V.; Sinitskii, A.; Sapoletova, N. A.; Napolskii, K. S.; Eliseev, A. A.; Byelov, D. V.; Grigorieva, N. A.; Vasilieva, A. V.; Bouwman, W. G.; Kvashnina, K.; Snigirev, A.; Grigoriev, S. V.; Petukhov, A. V. Langmuir 2009, 25, 10408-10412.

(12) Hilhorst, J.; Wolters, J. R.; Petukhov, A. V. CrystEngComm 2010, $12,3820-3826$.

(13) Taton, T. A.; Norris, D. J. Nature 2002, 416, 685-686.

(14) Masse, P.; Reculusa, S.; Clays, K.; Ravaine, S. Chem. Phys. Lett. 2006, 422, 251-255.

(15) Rinne, S. A.; Garcia-Santamaria, F.; Braun, P. V. Nat. Photonics 2008, 2, 52-56.

(16) Mau, S. C.; Huse, D. A. Phys. Rev. E: Stat. Phys., Plasmas, Fluids, Relat. Interdiscip. Top. 1999, 59, 4396-4401.

(17) Jiang, P.; Bertone, J. F.; Hwang, K. S.; Colvin, V. L. Chem. Mater. 1999, 11, 2132-2140.

(18) Vos, W. L.; Megens, M.; Van Kats, C. M.; Bosecke, P. Langmuir 1997, 13, 6004-6008.

(19) Thijssen, J. H. J.; Petukhov, A. V.; Van't Hart, D. C.; Imhof, A.; Van der Werf, C. H. M.; Schropp, R. E. I.; Van Blaaderen, A. Adv. Mater. 2006, 18, 1662-1666.

(20) Schöpe, H. J.; Barreira Fontecha, A.; Konig, H.; Marques Hueso, J.; Biehl, R. Langmuir 2006, 22, 1828-1838.

(21) Wang, Z. L.; Chan, C. T.; Zhang, W. Y.; Chen, Z.; Ming, N. B.; Sheng, P. Phys. Rev. E: Stat., Nonlinear, Soft Matter Phys. 2003, 67, No. 016612.

(22) Meng, L.; Wei, H.; Nagel, A.; Wiley, B. J.; Scriven, L. E.; Norris, D. J. Nano Lett. 2006, 6, 2249-2253.

(23) Van Blaaderen, A.; Wiltzius, P. Science 1995, 270, 1177-1179.

(24) Wei, H.; Meng, L.; Jun, Y.; Norris, D. J. Appl. Phys. Lett. 2006, 89.

(25) Hell, S. W. Nature 2007, 316, 1153-1158.

(26) Harke, B.; Ullal, C. K.; Keller, J.; Hell, S. W. Nano Lett. 2008, 8, 1309-1313.

(27) Wijnhoven, J. E. G. J.; Vos, W. L. Science 1998, 281, 802-804.

(28) Friedrich, H.; Gommes, C. J.; Overgaag, K.; Meeldijk, J. D.; Evers, W. H.; de Nijs, B.; Boneschanscher, M. P.; de Jongh, P. E.; Verkleij, A. J.; de Jong, K. P.; van Blaaderen, A.; Vanmaekelbergh, D. Nano Lett. 2009, 9, 2719-2724.

(29) Evers, W. H.; Friedrich, H.; Filion, L.; Dijkstra, M.; Vanmaekelbergh, D. Angew. Chem., Int. Ed. 2009, 48, 9655-9657.

(30) Gulden, J.; Yefanov, O. M.; Mancuso, A. P.; Abramova, V. V.; Hilhorst, J.; Byelov, D.; Snigireva, I.; Snigirev, A.; Petukhov, A. V.; Vartanyants, I. A. Phys. Rev. B: Condens. Matter Mater. Phys. 2010, 81, No. 224105

(31) Rodenburg, J. M.; Hurst, A. C.; Cullis, A. G.; Dobson, B. R.; Pfeiffer, F.; Bunk, O.; David, C.; Jefimovs, K.; Johnson, I. Phys. Rev. Lett. 2007, 98, No. 034801.

(32) Bosak, A.; Snigireva, I.; Napolskii, K. S.; Snigirev, A. Adv. Mater. 2010, 22, 3256-3259.
(33) Dierolf, M.; Menzel, A.; Thibault, P.; Schneider, P.; Kewish, C. M.; Wepf, R.; Bunk, O.; Pfeiffer, F. Nature 2010, 467, 436-440.

(34) Van Schooneveld, M. M.; Hilhorst, J.; Petukhov, A. V.; Tyliszczak, T.; Wang, J.; Weckhuysen, B. M.; De Groot, F. M. F.; De Smit, E. Small 2011, 7, 804.

(35) Stober, W.; Fink, A.; Bohn, E. J. Colloid Interface Sci. 1968, 26, 62-69.

(36) Philipse, A. P.; Vrij, A. J. Colloid Interface Sci. 1989, 128, 121136.

(37) Kaznatcheev, K. V.; Karunakaran, C.; Lanke, U. D.; Urquhart, S. G.; Obst, M.; Hitchcock, A. P. Nucl. Instrum. Methods 2007, 582, 9699.

(38) aXis2000 is free for noncommercial use. It is written in Interactive Data Language (IDL) and available online at http:// unicorn.mcmaster.ca/aXis2000.html.

(39) Kremer, J. R.; Mastronarde, D. N.; McIntosh, J. R. J. Struct. Biol. 1996, 116, 71-76.

(40) Obst, M.; Wang, J.; Hitchcock, A. P. Geobiology 2009, 7, 577591.

(41) Johansson, G. A.; Tyliszczak, T.; Mitchell, G. E.; Keefe, M.; Hitchcock, A. P. J. Synchrotron Radiat. 2007, 14, 395-402.

(42) Crocker, J. C.; Grier, D. G. J. Colloid Interface Sci. 1996, 179, 298-310.

(43) Wang, W.; Asher, S. A. J. Am. Chem. Soc. 2001, 123, 1252812535.

(44) Grigoriev, S. V.; Napolskii, K. S.; Grigoryeva, N. A.; Vasilieva, A. V.; Mistonov, A. A.; Chernyshov, D. Y.; Petukhov, A. V.; Belov, D. V.; Eliseev, A. A.; Lukashin, A. V.; Tretyakov, Y. D.; Sinitskii, A. S.; Eckerlebe, H. Phys. Rev. B: Condens. Matter Mater. Phys. 2009, 79, No. 045123.

(45) Braun, P. V.; Wiltzius, P. Adv. Mater. 2001, 13, 482-485.

(46) Mourad, M. C. D.; Devid, E. J.; Van Schooneveld, M. M.; Vonk, C.; Lekkerkerker, H. N. W. J. Phys. Chem. B 2008, 112, 10142-10152.

(47) Thibault, P.; Dierolf, M.; Menzel, A.; Bunk, O.; David, C.; Pfeiffer, F. Science 2008, 321, 379-382. 